\title{
Elongational-flow-induced scission of DNA nanotubes in laminar flow
}

\author{
Rizal F. Hariadi* \\ Department of Applied Physics, California Institute of Technology, Pasadena, California 91125, USA \\ Bernard Yurke \\ Materials Science and Engineering Department and Electrical and Computer Engineering Department, \\ Boise State University, Boise, Idaho 83725, USA \\ (Received 25 August 2009; revised manuscript received 2 May 2010; published 19 October 2010)
}

\begin{abstract}
The length distributions of polymer fragments subjected to an elongational-flow-induced scission are profoundly affected by the fluid flow and the polymer bond strengths. In this paper, laminar elongational flow was used to induce chain scission of a series of circumference-programmed DNA nanotubes. The DNA nanotubes served as a model system for semiflexible polymers with tunable bond strength and cross-sectional geometry. The expected length distribution of fragmented DNA nanotubes was calculated from first principles by modeling the interplay between continuum hydrodynamic elongational flow and the molecular forces required to overstretch multiple DNA double helices. Our model has no-free parameters; the only inferred parameter is obtained from DNA mechanics literature, namely, the critical tension required to break a DNA duplex into two single-stranded DNA strands via the overstretching B-S DNA transition. The nanotube fragments were assayed with fluorescence microscopy at the single-molecule level and their lengths are in agreement with the scission theory.
\end{abstract}

DOI: 10.1103/PhysRevE.82.046307

\section{INTRODUCTION}

Elongational-flow-induced scission can break a long polymer into fragments with controlled size and is an important physical technique in genome sequencing and biopolymer science [1]. Elongational-flow-induced scission of genomic DNA into controlled narrow distribution of short fragments, but with random break points, is a critical preparatory technique for producing unbiased DNA libraries in shotgun genome sequencing [2-4]. The fluid-flow-induced mechanical shearing of prion fibrils is routinely used in prion studies to replicate structural conformation of the determinant nuclei by generating new polymerizing ends $[5,6]$.

Polymer scission in a strong elongational flow occurs because of the interplay between macroscale hydrodynamic flows and atomic-scale intramolecular interactions of the polymer [7]. Substantial effort has been made toward understanding polymer scission, including elucidation of the scaling relations between key physical parameters $[3,8-14]$ and measurement of the polymer bond strength based on the fragment distributions. Recently, Vanapalli et al. reconciled the scaling discrepancies between theory and scission experiments and showed the significance of turbulent flow in polymer scission data [13].

Despite the amenability of laminar flow in the vicinity of a rigid rod to rigorous theoretical investigation, there have been no systematic studies of the scission of rigid polymers in the absence of turbulence. First, because of the weak elongational flow in the laminar regime, only long polymers on the order of a micron in length can accumulate enough tension for polymer scission to occur. Due to this requirement, polymer scission in laminar flow is considered "extraordinar-

\footnotetext{
*hariadi@dna.caltech.edu
}

PACS number(s): 47.15.-x, 87.15.Fh, 83.50.Jf, 62.25.-g

ily difficult" to achieve [13]. In our investigation, this long contour length challenge was satisfied by using long DNA nanotubes. This structure self-assembles cooperatively from individual $5-15 \mathrm{~nm}$ size components through a nucleation and condensation mechanism [15-17] that yields long tubular structures on the order of $5 \mu \mathrm{m}$. The second ramification from the weak induced tension in laminar flow is that for polymer scission to occur, the molecular forces between polymer subunits must be weak enough to be broken apart by the weak flow. In contrast to the polymer samples in previous scission studies, DNA nanotubes are held together by noncovalent interactions between their subunits. These two properties of DNA nanotubes, namely, long contour length and weak noncovalent intramolecular interactions, enable us to rigorously investigate polymer scission in laminar flow.

Here, we report the scission of circumferenceprogrammed DNA nanotubes in a purely laminar flow device. Scission is achieved when the tension along a DNA nanotube becomes sufficient to break the noncovalent basepair interactions holding the structure together. In our DNA nanotube construct, breakage is expected when the tension along individual duplex DNA strands is sufficient to induce a B-S transition from the B form of the double helix to the S form of the DNA overstretched state [18]. In a duplex DNA with two opposite nicks, the overstretching transition disrupts base pairings along the entire length of duplex DNA and allows the two strands to slide past each other until duplex DNA is completely melted into two free single-stranded DNAs. To generate quantifiable fluid flows with sufficient elongation rates, a syringe pump-driven microfluidic device was employed. The DNA nanotube fragment size distribution was quantified using single-molecule fluorescence microscopy. We derived a model without free parameters and validated the model predictions using the experimental data over nearly a decade of elongational flow rates and for DNA 


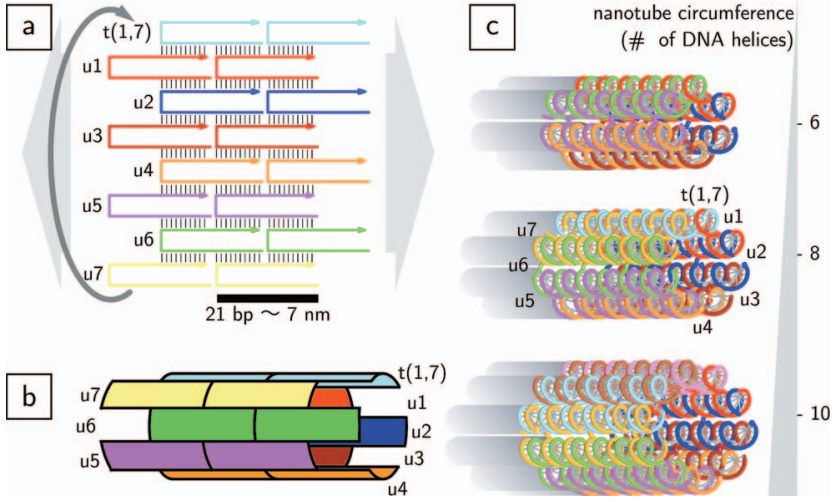

FIG. 1. (Color) An eight-helix nanotube is chosen to illustrate the modular construct of the DNA nanotube system used in this experiment adapted from [15]. (a) Complementarity graph of the eight-helix DNA nanotube. Each tile has four binding domains; each domain has a unique complement in its adjacent tile. The interaction between complementary domains drives the assembly into the designed order. (b) Each $t(1, n-1)$ strand concatenates two $u 1$ and two $u(n-1)$ strands and, thus wraps the two-dimensional crystalline structure into an $n$-helix nanotube. The same strategy has been demonstrated to successfully produce DNA nanotubes up to 20 duplex helices in circumference [15]. (c) Putative structures of six-, eight-, and ten-helix nanotubes.

nanotubes having three different tube circumferences and bond strengths.

\section{METHODS}

The DNA nanotubes used in this experiment are composed of recently devised "single-stranded-tile" structures [15]. These nanotube constructs are self-assembled structures that are rationally designed by encoding information in the sequence of DNA subunits using the techniques of structural DNA nanotechnology [19-22]. Single-stranded tile-based DNA nanotubes [15] represent a new variant of onedimensional crystalline DNA nanostructures as they are homogeneous in their circumferences. Current common model systems for semiflexible biopolymers, such as microtubules [23] and earlier DNA nanotube motifs [20,24], suffer from a circumference heterogeneity. Single-stranded tile-based DNA nanotubes can potentially serve as a controlled model system for semiflexible polymer physics due to their monodispersity and amenable physical properties, namely, circumference, bond strength, and persistence length.

In the single-stranded-tile construct, each 42-base DNA subunit binds to four of its neighbors with noncovalent basepair interactions [Fig. 1(a)]. Monodisperse $n$-helix nanotubes consist of $n$ unique DNA single-stranded subunits that selfassemble according to the complementarity graph shown in Fig. 1(a). Remarkably, the collective interaction between flexible single-stranded DNA subunits during lattice formation yields a tubular structure with uniform circumference and long contour length on the order of $5 \mu \mathrm{m}$ [15]. The DNA base sequence, crossover points [25], and location of nicks have translational symmetries along the longitudinal axis with periodicity of 21 base pairs $(\sim 7 \mathrm{~nm})$. The rupture

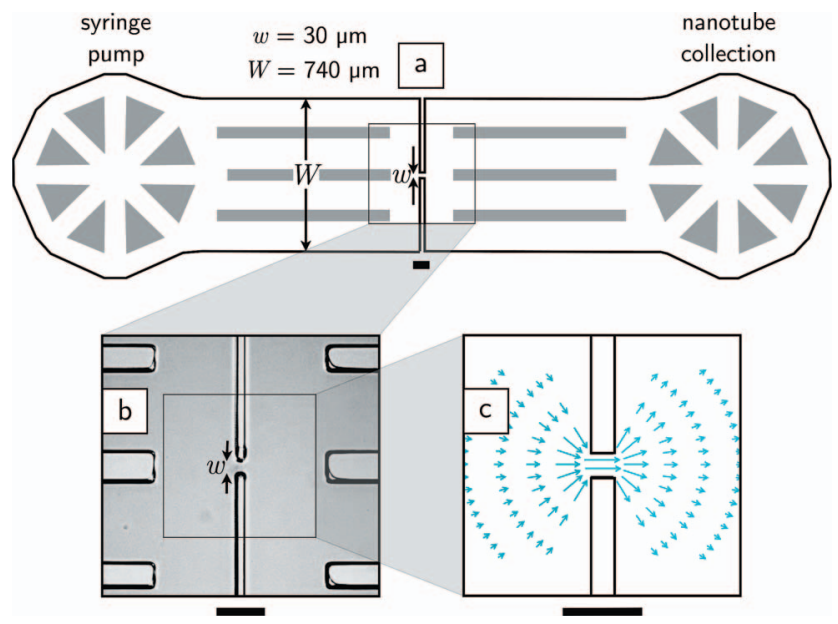

FIG. 2. (Color) (a) Schematic of the microfluidic chip used in the scission experiment. The nanotube sample was supplied via a syringe pump and collected in a vial before deposition on a microscope slide. (b) Light microscopy image of the microfluidic chamber used to produce the laminar elongational flow field. (c) Schematic of the putative streak lines of flow around the orifice. All scale bars are $100 \mu \mathrm{m}$.

is expected to occur when the drag force is sufficient to break a ring of $n$-DNA binding domains along the angular axis of the $n$-helix nanotube.

The persistence lengths of our DNA nanotubes were calculated to be on the order of $10 \mu \mathrm{m}$ based on the model described in Refs. [20,26]. This considerable rigidity to nanotube bending is likely to arise collectively from the electrostatic repulsion of charges and the steric interaction of chemical groups along a single DNA helix and between multiple parallel DNA helices. The single-stranded tile-based DNA nanotubes have persistence lengths that are three orders of magnitude longer than the persistence length of their single-stranded DNA subunits (that is, less than $5 \mathrm{~nm}$ [27]). More importantly, these persistence lengths are longer than their average nanotube lengths, that is, on the order of $5 \mu \mathrm{m}$. Note also that in a different DNA nanotube construct [20], the mean and variance of nanotube length have been observed to increase over time due to end-to-end joining [24]. In hydrodynamic flow analysis, the substantial persistence length allows the treatment of the DNA nanotubes as rigid rods and allows us to neglect polymer vibrations.

The DNA nanotubes were prepared by mixing an equimolar subunit concentration $(\leq 3 \mu \mathrm{M})$ of $n$-programmed single-stranded DNA subunits (purchased from Integrated DNA Technologies, Inc.) in $1 \times \mathrm{TAE}(40 \mathrm{mM}$ trisacetate and

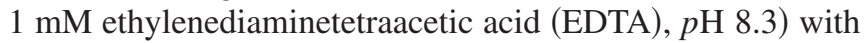
$12.5 \mathrm{mM} \mathrm{Mg}$-acetate $\cdot 4 \mathrm{H}_{2} \mathrm{O}$ and then slowly annealing from $90{ }^{\circ} \mathrm{C}$ to room temperature over the course of a day in a styrofoam box. For fluorescence imaging, a Cy3 fluorophore is covalently linked into the $5^{\prime}$ end of the single-stranded DNA subunit $u 1$ [see Fig. 1(a)], which corresponds to one fluorophore every $\sim 7 \mathrm{~nm}$ along the DNA nanotube.

The polydimethylsiloxane (PDMS) microfluidic device $[28,29]$ produces high elongational flow at the transition volume between a wide channel and a small orifice (Fig. 2). The width of the wide channel $W$ is $740 \mu \mathrm{m}$ and the orifice has 

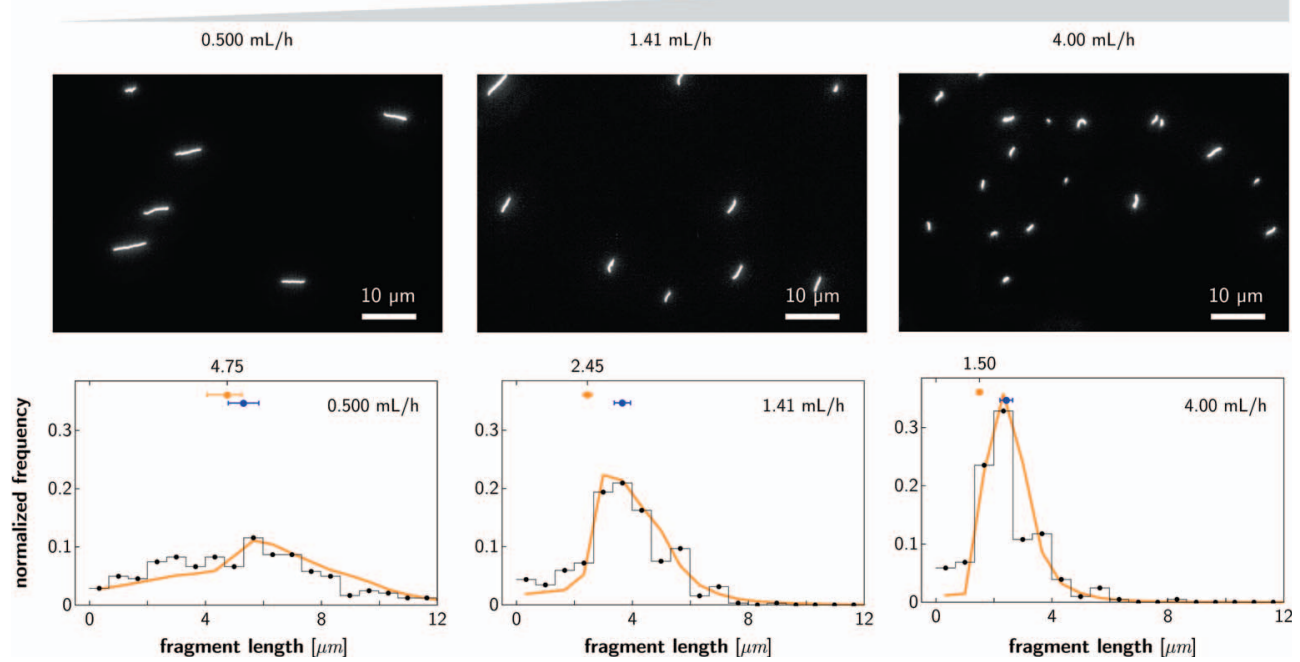

FIG. 3. (Color) Light microscopy images and fragment length histogram of eight-helix nanotubes after being subjected to volumetric flow rates at $0.500,1.41$, and $4.00 \mathrm{ml} / \mathrm{h}$. The mean fragment length and the Bayesian fit results are summarized in Table I. The orange solid line is the best Bayesian fit of the experimental data. The blue and orange dots with error bars are the average fragment length and most probable inferred $L_{\text {crit }}$, respectively.

a rectangular cross section with a width $w$ of $30 \mu \mathrm{m}$. We estimate that the width of the orifice is larger than the length of $84 \%$ of the DNA nanotubes in the test tube. The channel height $h$ is $20 \mu \mathrm{m}$ throughout the microfluidic chip.

Near the orifice, the flow is a laminar elongational flow [Fig. 2(c)]. At the microfluidic device entrance [labeled syringe pump in Fig. 2(a)], a capillary tube feeds the DNA nanotubes into the flow channel. In this region, the nanotubes are subjected to a much weaker elongational flow than in the area close to the orifice. This weak elongational flow is useful for preconditioning DNA nanotubes into a stretched conformation before entering the zone with high elongational flow. A control experiment involving a microfluidic chip without an orifice shows no detectable difference between the length distributions before (in the test tube) and after being subjected to the control microfluidic device. The large rectangular and triangular posts [gray-shaded regions in Fig. 2(a)] were required to prevent chamber deformation due to the elastomeric nature of PDMS and the relatively high pressures used in the scission experiments. Based on the comparison between dimensions of our device and the initial distribution of the DNA nanotubes, we claim that the presence of the posts does not perturb the flow pattern in the vicinity of the orifice where the scission occurs.

The upper bound on the range of flow rates investigated is given by the maximum rate at which the syringe pump can inject fluid into the microfluidic device without resulting in noticeable deformation and mechanical failure of the device. The minimum flow rate required to break a substantial fraction of the DNA nanotubes sets the lower limit on the range

TABLE I. The most probable $L_{c r i t}$ and mean fragment length for six-, eight-, and ten-helix nanotubes after scission at flow rate chosen to be powers of $\sqrt{2}$ in $\mathrm{ml} / \mathrm{h}$. For $L_{\text {crit }}$, the first number is the most probable value and the second and third entries are the lower and upper bounds of the $90 \%$ confidence interval. The mean is the mean fragment length of the sample. The uncertainty of the mean length is the standard deviation as determined by a bootstrapping technique.

\begin{tabular}{|c|c|c|c|c|c|c|c|c|c|}
\hline \multirow[b]{2}{*}{$\begin{array}{l}\text { Volumetric flow rate } \\
(\mathrm{ml} / \mathrm{h})\end{array}$} & \multicolumn{3}{|c|}{ Six-helix nanotube } & \multicolumn{3}{|c|}{ Eight-helix nanotube } & \multicolumn{3}{|c|}{ Ten-helix nanotube } \\
\hline & & $\begin{array}{l}L_{c r i t} \\
(\mu \mathrm{m})\end{array}$ & $\begin{array}{l}\text { Mean } \\
(\mu \mathrm{m})\end{array}$ & & $\begin{array}{l}L_{c r i t} \\
(\mu \mathrm{m})\end{array}$ & $\begin{array}{l}\text { Mean } \\
(\mu \mathrm{m})\end{array}$ & & $\begin{array}{l}L_{c r i t} \\
(\mu \mathrm{m})\end{array}$ & $\begin{array}{l}\text { Mean } \\
(\mu \mathrm{m})\end{array}$ \\
\hline$\sqrt{2^{-2}}=0.500$ & 3.95 & {$[3.65,4.50]$} & $4.40 \pm 0.22$ & 4.75 & {$[4.05,5.25]$} & $5.31 \pm 0.48$ & 4.85 & {$[4.25,5.25]$} & $5.87 \pm 0.22$ \\
\hline$\sqrt{2^{-1}} \approx 0.707$ & 3.00 & {$[2.80,3.25]$} & $4.19 \pm 0.43$ & 3.95 & {$[3.60,4.15]$} & $5.35 \pm 0.53$ & 4.20 & {$[3.90,4.95]$} & $5.47 \pm 0.51$ \\
\hline$\sqrt{2^{0}}=1.00$ & 2.70 & {$[2.45,2.85]$} & $3.91 \pm 0.38$ & 2.80 & {$[2.65,2.95]$} & $4.10 \pm 0.48$ & 3.70 & {$[3.45,3.95]$} & $5.10 \pm 0.46$ \\
\hline$\sqrt{2^{1}} \approx 1.41$ & 2.10 & {$[1.95,2.20]$} & $3.13 \pm 0.34$ & 2.45 & {$[2.30,2.60]$} & $3.65 \pm 0.37$ & 2.70 & {$[2.50,2.85]$} & $4.04 \pm 0.43$ \\
\hline$\sqrt{2^{2}}=2.00$ & 1.80 & {$[1.70,2.05]$} & $3.06 \pm 0.28$ & 2.15 & {$[1.90,2.25]$} & $3.18 \pm 0.28$ & 2.20 & {$[2.05,2.30]$} & $3.58 \pm 0.34$ \\
\hline$\sqrt{2^{3}} \approx 2.83$ & 1.50 & {$[1.40,1.60]$} & $2.51 \pm 0.27$ & 1.80 & {$[1.65,1.95]$} & $2.90 \pm 0.28$ & 1.75 & {$[1.65,1.90]$} & $2.95 \pm 0.30$ \\
\hline$\sqrt{2^{4}}=4.00$ & 1.45 & {$[1.35,1.55]$} & $2.29 \pm 0.22$ & 1.50 & {$[1.40,1.60]$} & $2.43 \pm 0.26$ & 1.65 & {$[1.55,1.75]$} & $2.71 \pm 0.25$ \\
\hline Control (device without the orifice) & & & $5.70 \pm 0.45$ & & & $6.32 \pm 0.31$ & & & $6.03 \pm 0.29$ \\
\hline
\end{tabular}




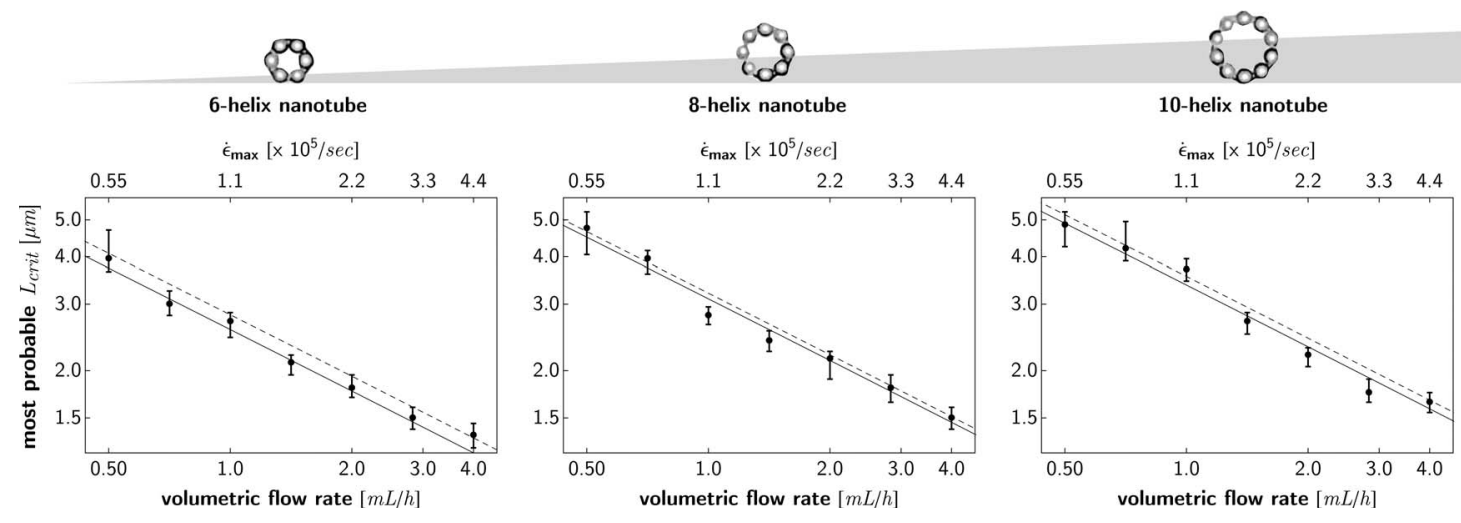

FIG. 4. Fragment length as a function of volumetric flow rate of six-, eight-, and ten-helix nanotubes. The solid line corresponds to the most probable of $L_{c r i t}$ from all data based on our theoretical model by Bayesian a priori probability. The dashed line is the theoretical curve with $f_{c}=65 \mathrm{pN}$ [18]. Note that for the same volumetric flow rate, the most probable $L_{c r i t}$ (solid black circle) increases with larger nanotube circumference.

of flow rates used in the reported experiments.

\section{RESULTS AND DISCUSSIONS}

In a scission experiment, a dilute DNA nanotube solution at $\sim 1 \mathrm{nM}$ initial tile concentration was injected into the microfluidic device at rates in the range of $0.500-4.00 \mathrm{ml} / \mathrm{h}$ using an automatic syringe pump. We found that the syringe pump is a better injection method than pressurized gas because of the absence of initial dead volume that slows down the initial volumetric flow rate. Each nanotube was passed into the microfluidic chamber only once. The first $50 \mu \mathrm{l}$ sample was discarded to avoid any contamination from the previous run and to make sure that the volumetric rate was constant during the scission of the collected sample. Without stopping the syringe pump, the next $20 \mu \mathrm{l}$ sample of fragmented DNA nanotubes was collected at the outlet port in a $500 \mu \mathrm{l}$ vial. A $5 \mu \mathrm{l}$ drop of this DNA solution was deposited between a cleaned microscope slide and a coverslip and placed on the microscope sample stage. The microscope slide and a coverslip cleaning procedure in Ref. [30] was followed. The presence of divalent cations in the buffer facilitates the formation of salt bridges between the two negatively charged species, namely, the DNA fragments and the glass surface. Once the DNA nanotubes were immobilized on the glass coverslip, any further reactions, such as end-toend joining, spontaneous scission [20,24], and polymerization, are quenched. Thus, the images are the record of the fragment distribution immediately after being subjected to the elongational flow.

The nanotube fragment distribution was imaged with a home-built total internal reflection fluorescence microscope as previously described in [15] and quantified at the singlemolecule level with ImageJ [31]. The number of photons emitted by a DNA nanotube was used as a proxy for nanotube length. In each frame, the longest nanotube whose length could be easily measured provided a calibration for this proxy. This technique is insensitive to the curvature of DNA tubes and how focused each fragment image is. Moreover, the photon-counting method allows for the determina- tion of nanotube lengths even for fragments that are not optically resolved. The single-molecule assay enables us to exclude experimental artifacts resulting from the rare occurrence of high mass nanotube aggregates which were visually identified and not counted. Nanotube aggregation is expected to behave differently in elongational flow, leading to different fragment size distributions than for pristine DNA nanotubes. All features whose maximum pixel intensities were above the saturation level of the camera were excluded from the length measurement.

In Fig. 3 (top row), we show snapshots of eight-helix nanotube fragments imaged immediately after a scission experiment at $0.500,1.41$, and $4.00 \mathrm{ml} / \mathrm{h}$ volumetric flow rates $\dot{V}$. The Reynolds number Re for the fluid flow within the orifice, at the fastest volumetric flow rates used, was calculated to be 25 , which is safely within the laminar regime $(\operatorname{Re}<2000)$. Elsewhere in the system, the fluid velocities and the corresponding Re are smaller. The light microscopy images and the corresponding length histograms show the

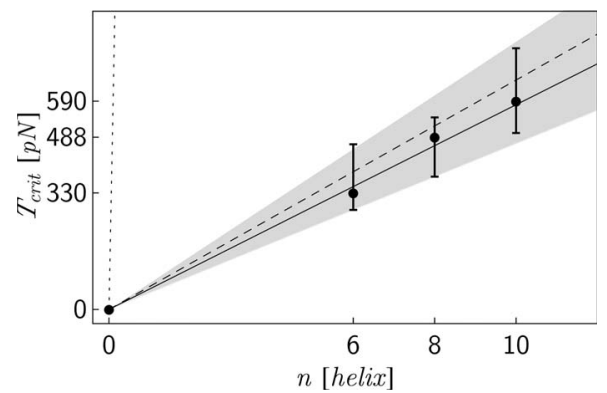

FIG. 5. Inferred $T_{\text {crit }}$ as a function of nanotube circumference. The solid and dashed lines correspond to the most probable $\left(f_{c}\right.$ $=58 \mathrm{pN})$ and the literature value of the critical tension required $\mathrm{B}-\mathrm{S}$ DNA overstretching transition $\left(f_{c}=65 \mathrm{pN}\right)$ [18], respectively. The linear fit was constrained to intersect the point of origin $(0,0)$. The gray region represents the $90 \%$ confidence area for the linear fit that passes through the point of origin. The steep dotted line illustrates the critical strength of breaking covalent bonds in DNA backbones $\left(f_{c}=2 n \times 5860 \mathrm{pN}\right)[13,14,34]$, which has a much steeper slope than our experimental data. 


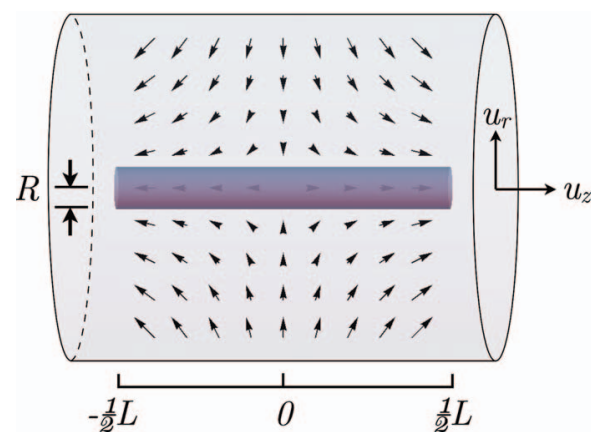

FIG. 6. (Color) A rigid rod in an axially symmetric elongational flow.

dependence of fragment size on volumetric flow rate. Faster flow rates generate higher elongational rates and shorter fragment size (Fig. 3). The same experiment was repeated with DNA nanotubes having different circumferences and corresponding bond strengths, namely, the six- and ten-helix nanotubes, and the same trend was consistently observed in all nanotubes (see Table I and Figs. 4, 7, and 8).

Elongational flow induces the alignment of DNA nanotubes along the flow gradient. According to the scission theory presented in Appendix A, the drag force experienced by the nanotubes induces tension along the axis of the DNA nanotubes. This tension is greatest at the midpoint of DNA nanotubes [32], and when it exceeds the tensile strength of the nanotube, the tube fragments into two shorter tubes of approximately equal length. In our microfluidic device, the elongational rate is proportional to the reciprocal of the square of the distance to the orifice $1 / \rho^{2}$ [Eq. (A13)]. Hence, after encountering an elongation flow regime sufficient to break the nanotube in two, the fragments may encounter a flow regime which is sufficient to break each newly generated fragment again into two shorter fragments of approximately equal length. This process of scission will continue until the length of the individual fragment is such that the tensions exerted in the region of highest elongational flow are insufficient to result in chain scission. In our model, $2 L_{c r i t}$ is defined as the length of the shortest DNA nanotube that can be broken in two in the region of the highest elongational flow $\dot{\epsilon}_{\text {max }}$. Therefore, $L_{c r i t}$ is the length of the shortest DNA nanotube that can be produced by each elongational-flow-induced scission in our device at a particular volumetric flow rate. For a tube $i$ of length $L_{i}$, the number of scission rounds is given by $m_{i}=\left\lfloor\ln \left(L_{i} / L_{\text {crit }}{ }_{i}\right) / \ln (2)\right\rfloor$, where the brackets denote rounding off to the nearest integer (Appendix B). In our model, an initial tube $i$ of length $L_{i}$ yields $2^{m_{i}}$ output fragments that have identical length of $L_{i} /\left(2^{m_{i}}\right)$.

We employed stochastic scission simulation and Bayesian inference (Appendix B) to extract $L_{c r i t}$ from each fragment histogram data $\mathcal{H}$. The mean fragment length is not a valid estimate for $L_{c r i t}$ because the mean of the fragment length distribution is affected by the DNA nanotube distribution before being subjected to the elongational flow. The Bayesian inference has to include the stochasticity of the scission events in our device. The elongational flow in the device and the flux of the DNA nanotube are not uniform but are functions of position $\left(x_{i}, y_{i}\right)$ of DNA nanotube $i$ within the chan- nel. In particular, they will be zero at the channel walls and maximum at the center of the channel. Hence, even if we start with a population of DNA nanotubes that is monodisperse in size, the length of the DNA nanotube fragments produced will be different at different points within the orifice.

The results of the Bayesian inference of $L_{c r i t}$ are presented in Figs. 3 and 4, Table I, and Appendix C. Table I lists the most probable $L_{\text {crit }}$, its $90 \%$ probability interval, and the mean DNA nanotube length for six-, eight-, and ten-helix nanotubes for various fluid-flow rates. In Fig. 3 and also in Fig. 7 of Appendix $C$, the orange circle represents the most probable $L_{c r i t}$ and the orange error bar is the range where the a posteriori probability is over $90 \%$. For comparison, the mean fragment lengths and their uncertainties are indicated in blue. As expected, the difference between fragment mean and $L_{\text {crit }}$ is less significant when $L_{\text {crit }}$ approaches the initial fragment mean [Fig. 3 (left panel)] because in that regime the elongational flow breaks only an insignificant portion of the initial nanotubes. The Bayesian inference performs poorly when $L_{\text {crit }}$ approaches the mean of control nanotube distribution, as illustrated by the wider $90 \%$ confidence bands in Table I and longer error bar in Fig. 3 (and also in Fig. 7 of Appendix C) for the slowest volumetric flow rate. The Bayesian inferred $L_{c r i t}$ of the slowest volumetric flow rate experiment might be still very good, but the data do not warrant strong conclusion.

The most probable inferred $L_{c r i t}$ is plotted against the volumetric flow rate in Fig. 4. For comparison, the no-freeparameter theoretical prediction of Eq. (A15) using $f_{c}$ $=65 \mathrm{pN}$ is shown as a dashed line in the figures, where $f_{c}$ is the critical tension required to overstretch a single DNA double helix $[18,33]$. The theoretical line has a slope of -0.5 in these double-logarithmic plots, indicating that $L_{c r i t}$ scales as the square root of the flow rate. Linear fitting of the Bayesian inferred $L_{\text {crit }}$ with respect to volumetric flow rate yields the slope to be $-0.52 \pm 0.06,-0.55 \pm 0.07$, and $-0.57 \pm 0.07$ for six-, eight-, and ten-helix nanotubes, respectively. In all measured nanotubes, the theoretical exponent is within the $90 \%$ confidence interval of our linear fit, giving us confidence in the -0.5 theoretical scaling of $L_{c r i t}$ with the volumetric flow rate or with the elongational rate. The $L_{c r i t} / 2 R$ term in Eq. (A15) is on the order of $10^{2}$ and its natural logarithm was treated as constant and absorbed by the fitted slope in the linear fit for each DNA nanotube circumference.

Having established confidence in the scaling based scission theory, we use Eq. (A15) in a separate Bayesian inference to obtain an experimental value of the tension required to simultaneously break $n$ parallel DNA helices $T_{c r i t}=n f_{c}$ [Eq. (A11)]. In this Bayesian inference, the fit takes into account the probability $\operatorname{Prob}\left(L_{\text {crit }} \mid \mathcal{H}\right)$ at various volumetric flow rates. Separate inference analysis of each nanotube yields 330,488 , and $590 \mathrm{pN}$ as the most probable bond strength of six-, eight-, and ten-helix nanotubes. The $90 \%$ confidence bands span across 282-468, 376-544, and 500$740 \mathrm{pN}$ for six-, eight-, and ten-helix nanotubes, respectively. In Fig. 5, the linear trend of the inferred $T_{\text {crit }}$ as a function of the number of DNA double helices in the tube circumference is in agreement with Eq. (A11).

Finally, to extract an experimental $f_{c}$, we perform a Bayesian inference on all the data, imposing the -0.5 scaling re- 

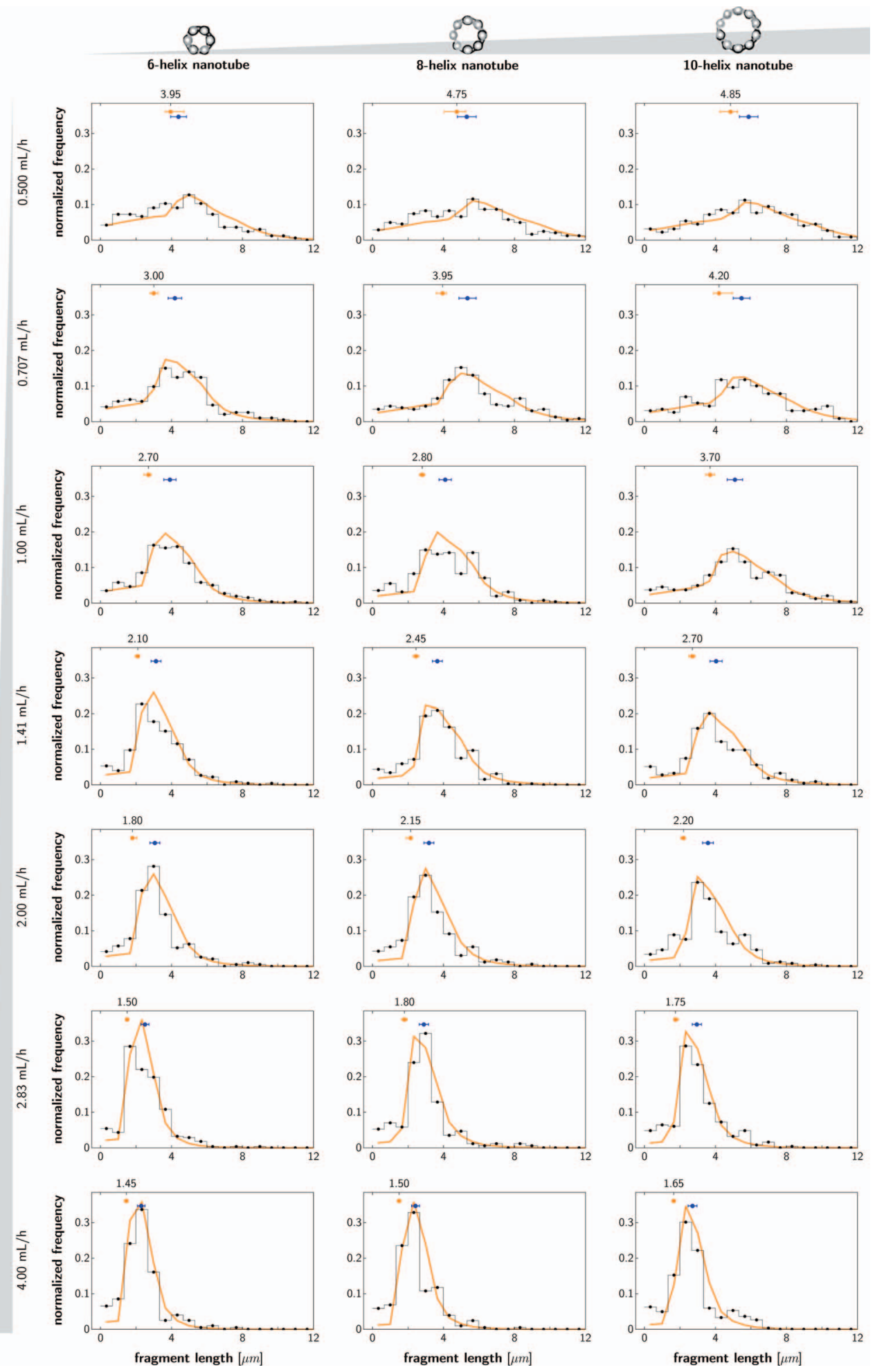

FIG. 7. (Color) Best $L_{\text {crit }}$ fit by Bayesian inference (see Appendix C for details).

lation between $L_{c r i t}$ and flow rate and the linear scaling of $T_{c r i t}$ with $n$. The most probable $f_{c}$ was inferred to be $58 \mathrm{pN}$, with a $90 \%$ confidence band spanning across $47-76 \mathrm{pN}$. Our measurement is consistent with the reported $45-65 \mathrm{pN}$ as the applied tension when overstretch transition occurs in various experimental conditions, namely, ionic concentration and temperature $[18,33]$. All of our scission experiments were performed at room temperature. The striking agreement further validates our scission model and its assumption that all the DNA helices contribute to the total bond strength cooperatively as assumed in our model. The measured critical tension is consistent with the notion that the elongationalflow-induced tension breaks the noncovalent interactions, and the DNA nanotube scission occurs via a collective B-S 


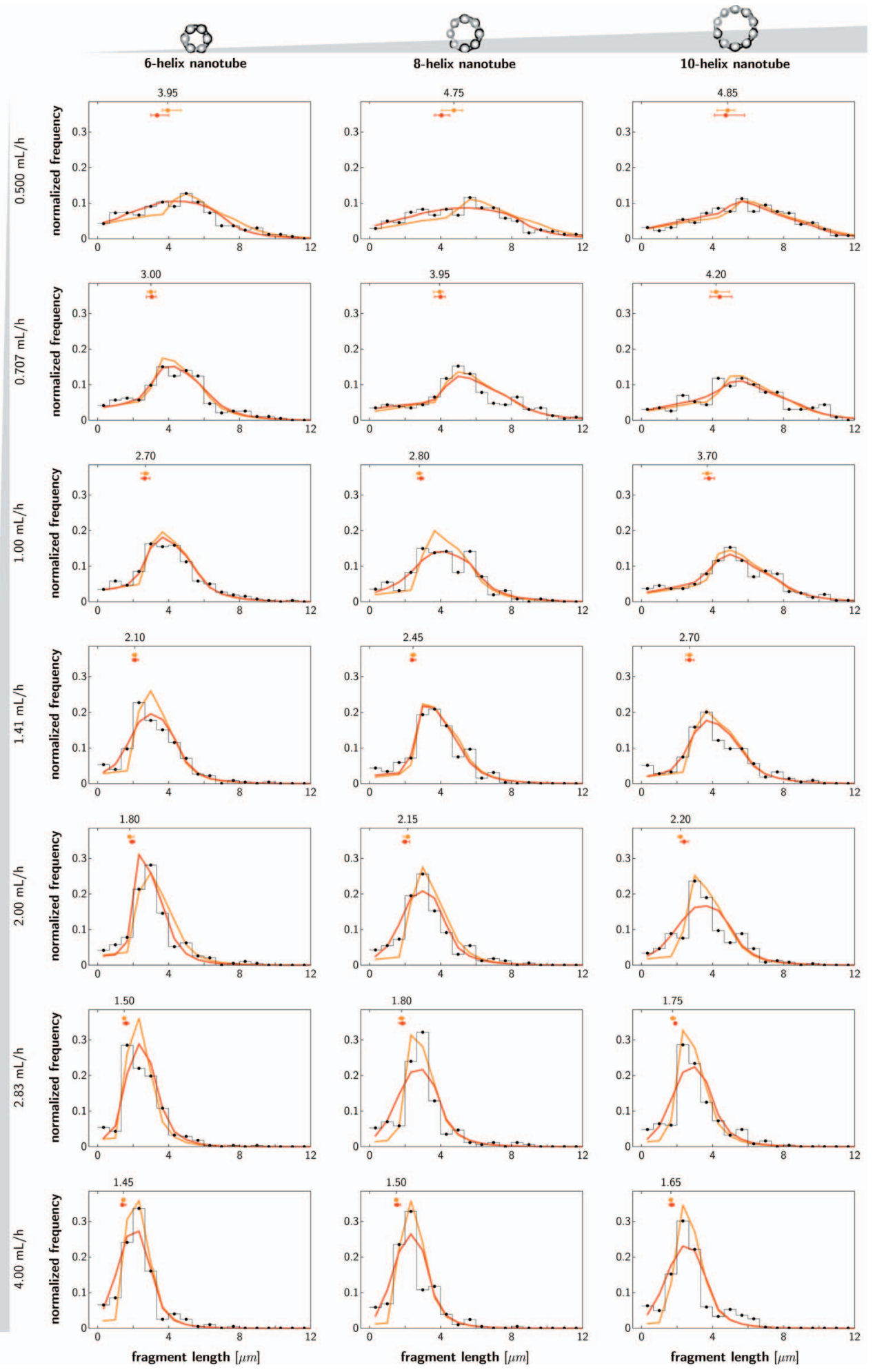

FIG. 8. (Color) Best $L_{\text {crit }}$ fit by Bayesian inference with truncated Gaussian noise (see Appendix D for details). The red lines are the Bayesian fits with noise.

transition from the $\mathrm{B}$ form of double helices to the $\mathrm{S}$ form of the overstretched state of DNAs at the breaking point. We note that the bond strength value for breaking a covalent phosphodiester bond in the DNA backbone was measured and calculated to be on the order of $5 \times 10^{3} \mathrm{pN}[13,14,34]$, which is approximately two orders of magnitude larger than the measured $f_{c}$ in this work (see Fig. 5).

That $f_{c}$ should be the force required to overstretch DNA is based on the notion that passage through the region of high elongational flow is fast compared to the time scale which 
would allow the DNA tubes to break apart by slower, less energetic, relaxation mechanisms, such as those involving thermal fluctuations and base-pair breathing. In particular, the transit time of the DNA through the region of high elongational flow in our microfluidic device ranges from 7 to $60 \mu \mathrm{s}$ for the fastest and slowest flow rates used in this experiment, respectively. These times are comparable to the $10 \mu$ s that it takes for a branch point to move by one base position in three-strand branch migration [35,36]. We note that the time scale involving rearrangement of a few bases is already comparable to the transit times of the high flow region near the orifice (Fig. 2(c)).

It is conceivable that each midpoint scission event will produce two fragments that are not exactly equal in length. Based on our theory in Appendix A [Eq. (A10)], the distribution of tension along the nanotube is approximately parabolic, being maximum at the midpoint and symmetrically dropping to zero at both ends. Thus, the applied tension reaches a plateau at the center of the fragment in which the scission could occur anywhere due to unmodeled physical sources of randomness while still maintaining its midpoint as the most probable location for scission.

In order to evaluate the effect of randomness in our experiment, we incorporated tunable truncated Gaussian noise into the previously presented Bayesian inference to account for other plausible sources of randomness that are unmodeled in our theory (see Appendix D). The tunable parameters in this new Bayesian fit are $L_{\text {crit }}$ and the standard deviation of the truncated Gaussian noise $\sigma_{i}$ relative to the nanotube length $L_{i}$. Excluding the slowest volumetric flow rate result, the most probable $L_{c r i t}$ from Bayesian inference by a posteriori probability from the same model with various Gaussian noise added agrees with the most probable $L_{\text {crit }}$ from inference with our simple scission theory within 5\%. This insight leads us to conclude that the noise has to be implausibly large to make a noticeable difference in our inference and that the assumption of the absence of other plausible physical factors which could contribute to noise in the theoretical model and Bayesian inference is justified.

\section{CONCLUDING REMARKS}

In this paper, we presented the results of systematic experiments on the scission of DNA nanotubes with welldefined circumferences in a microfluidic device with a welldefined region of laminar elongational flow. This allowed us to rigorously test the scission theory involving no adjustable parameter, presented in Appendix A. We find that the theory accurately predicts DNA nanotube fragment size as a function of elongational rate and the number of circumferential helices of the tube. Since fragment size is a predictor of the maximum elongation rate encountered by a DNA nanotube, we suggest that DNA nanotubes can be used as microscopic probes to measure the maximum elongation rates encountered in fast, small-scale, or complex hydrodynamic flow fields [37].

\section{ACKNOWLEDGMENTS}

We are indebted to Erik Winfree for generously hosting this work in his lab and for his valuable and insightful input to the project. The authors would like to thank Rebecca Schulman, Peng Yin, Damien Woods, Victor A. Beck, Elisa Franco, Zahid Yaqoob, Karthik Sarma, Saurabh Vyawahare, Nadine Dabby, Tosan Omabegho, Jongmin Kim, Imran Malik, and Michael Solomon for valuable discussions. It is our great pleasure to acknowledge the support of the NASA Astrobiology Grant No. NNG06GAOG, NSF Grants No. DMS0506468, No. EMT-0622254, and No. NIRT-0608889, and the Caltech Center of Biological Circuit Design grants. This work was initiated by a serendipitous observation of elongational-flow-induced fragmantation of DNA nanotubes by Harry M. T. Choi and was undertaken to facilitate the design of a fluidics system for Rebecca Schulman and Erik Winfree's project on engineering DNA tile-based artificial life [38]. The DNA nanotubes used in this experiment and their three-dimensional illustrations were generous gifts from Peng Yin. The design and manufacturing process of the PDMS microfluidic chip were assisted by the Caltech Microfluidic Foundry.

\section{APPENDIX A: UNDERLYING SCISSION THEORY}

Here, we present the hydrodynamic model used for comparison with our experiment. First, we derive an expression for the tension produced at the midpoint of a long cylinder. Then, an expression for the maximum elongation rate $\dot{\epsilon}$ for the microfluidic device is obtained.

In the approach taken to determine the tension produced at the midpoint of a long cylinder, the exact solution for fluid flow in the presence of a cylinder of infinite length is approximately matched with an exact solution for axially symmetric elongational flow in the absence of the rod (Fig. 6).

For the case of low Reynolds number flow in an incompressible fluid, the continuity equation and the Navier-Stokes equations are reduced to

$$
\begin{gathered}
\boldsymbol{\nabla} \cdot \mathbf{u}=0, \\
\nabla P=\mu \nabla^{2} \mathbf{u},
\end{gathered}
$$

where $\mathbf{u}$ is the velocity field, $P$ is the pressure, and $\mu$ is the viscosity. From these two equations, it follows that $\nabla^{2} P=0$. One can verify by direct substitution that

$$
u_{r}=\frac{C}{2 \mu}\left[r \ln \left(\frac{r}{R}\right)-\frac{r}{2}+\frac{R^{2}}{2 r}\right]
$$

and

$$
u_{z}=-\frac{C}{\mu} \ln \left(\frac{r}{R}\right) z
$$

which are exact solutions of Eqs. (A1) and (A2), where $C$ and $R$ are integration constants. The solution represents a fluid flow around a cylinder of radius $R$ of infinite extent for no-slip boundary conditions that is evident from the fact that the fluid velocity vanishes at $r=R$.

The fluid velocity field without the rod representing axially symmetric fluid flow with an elongational rate of $\dot{\epsilon}$ along the $z$ axis is given by 


$$
\begin{gathered}
u_{r}=-\frac{\dot{\epsilon}}{2} r, \\
u_{z}=\dot{\epsilon} z,
\end{gathered}
$$

as can be verified by direct substitution into Eqs. (A1) and (A2). Since the first term of Eq. (A3) dominates when $r$ is large, a good approximate match between the solution given by Eqs. (A3) and (A4) and that of Eqs. (A5) and (A6) at the characteristic crossover distance $r=L / 2$ is obtained by setting

$$
C=-\frac{\mu \dot{\epsilon}}{\ln (L / 2 R)},
$$

where $R$ and $L$ are the radius and the length of $n$-helix DNA nanotube, respectively. Equation (A4) then becomes

$$
u_{z}=\dot{\epsilon} \frac{\ln (r / R)}{\ln (L / 2 R)} z \text {. }
$$

The flow induced stress in the $z$ direction on the cylinder's surface is given by

$$
\left.\sigma_{r z} \equiv \mu \frac{\partial u_{z}}{\partial r}\right|_{r=R}=\frac{\mu \dot{\epsilon} z}{R \ln (L / 2 R)} .
$$

The line tension at the center of the cylinder is thus given by

$$
T=4 \pi R \int_{0}^{L / 2} \sigma_{r z} d z=\frac{\pi \mu \dot{\epsilon} L^{2}}{4 \ln (L / 2 R)} .
$$

This expression is similar to the recently published expression of the elongational-flow-induced drag force in Ref. [13]. In our work [Eq. (A10)], we provide a derivation of the $\mathrm{O}(1)$ geometric constant for our device.

The scission occurs when the midpoint tension $T$ is larger than the critical tension required breaking all DNA helices simultaneously across the nanotubes. This critical tension is expected to be given by

$$
T_{c r i t}=n f_{c},
$$

where $n$ is the nanotube circumference and $f_{c}$ is the tension required to break a single DNA helix. In the DNA nanotubes, the DNA helices are aligned along the axis of the tube. Tension is thus exerted along the length of the binding domains of the participating DNA strands. One expects these binding domains to fail when the tension along the binding domains is greater than required to overstretch a DNA helix [18]; that is, one expects $f_{c}$ to be close to $65 \mathrm{pN}$.

In our device geometry, the flow into the narrow channel is approximately radial. We take the mean flow velocity (averaged over height) $\bar{u}$ to be given by

$$
\bar{u}(\rho)=-\frac{\overline{u_{w}} w}{\pi \rho},
$$

where $\rho$ is the radial distance to the channel entrance and $\overline{u_{w}}$ is the mean flow velocity across the orifice.

The elongational flow (averaged over height) $\overline{\dot{\epsilon}}$ is defined as

$$
\overline{\dot{\epsilon}} \equiv \frac{\partial \bar{u}}{\partial \rho}=\frac{\overline{u_{w}} w}{\pi \rho^{2}} .
$$

The elongational flow $\dot{\epsilon}$ is maximum near the orifice where $\pi \rho=w$,

$$
\left.\overline{\dot{\epsilon}}_{\max } \equiv \frac{\partial \bar{u}}{\partial \rho}\right|_{\rho=w / \pi}=\frac{\pi \overline{u_{w}}}{w}=\frac{\pi \dot{V}}{w^{2} h},
$$

where $\dot{V}$ is the volumetric flow rate of our syringe pump and is equal to $\overline{u_{w}}$ multiplied by the orifice cross-sectional area.

In Fig. 4 , the theoretical prediction of $L_{c r i t}$ for scission experiment of $n$-helix nanotube over a range of $\dot{V}$ is obtained by setting $L=L_{\text {crit }}, T=T_{\text {crit }}$, and $\dot{\epsilon}=\dot{\epsilon}_{\text {max }}$ and substituting Eqs. (A11) and (A14) to Eq. (A10) that yields the equation below,

$$
T_{\text {crit }}=\frac{\pi \mu \dot{\epsilon}_{\text {max }} L_{c r i t}^{2}}{4 \ln \left(L_{c r i t} / 2 R\right)},
$$

where $T_{\text {crit }}$ is given by Eq. (A11) and $\dot{\epsilon}_{\text {max }}$ is elongational flow at the center of the channel and at a distance of $\rho$ $=w / \pi$ from the orifice where the maximum elongation flow is expected to occur.

Note that the radial flow approximation in Eq. (A12) is only valid for a point far away from the constriction. Our scission model calculates the location of all scission events in all of our experiments to be at a distance $\rho>w / \pi$ from the orifice in order to produce the observed mean fragment length from the initial DNA nanotube distribution. This calculation is consistent with the expected position of $\overline{\dot{\epsilon}}_{\text {max }}$ in Eq. (A14) and the calculated $\overline{\dot{\epsilon}}$ profiles in similar constriction devices reported in $[39,40]$. Therefore, the radial flow approximation in Eq. (A12) is justified.

\section{APPENDIX B: BAYESIAN INFERENCE AND STOCHASTIC SCISSION SIMULATION}

In our data analysis, we utilized a Bayesian inference method to extract $L_{c r i t}$ out of the fragment length histogram data $\mathcal{H}$ by calculating the a posteriori probability $\operatorname{Prob}\left(L_{\text {crit }} \mid \mathcal{H}\right)=\operatorname{Prob}\left(\mathcal{H} \mid L_{\text {crit }}\right) \operatorname{Prob}\left(L_{\text {crit }}\right) / \operatorname{Prob}(\mathcal{H})$, where the a priori $\operatorname{Prob}\left(L_{c r i t}\right)$ is taken to be uniform over $0 \leq L_{\text {crit }}$ $\leq 10 \mu \mathrm{m}$ and zero otherwise. The upper bound is approximately twice the average nanotube length in the control experiment. $\operatorname{Prob}(\mathcal{H})$ is treated as a normalization constant and is set by constraining $\Sigma \operatorname{Prob}\left(L_{\text {crit }} \mid \mathcal{H}\right)=1 \operatorname{Prob}\left(\mathcal{H} \mid L_{\text {crit }}\right)$ was calculated by assuming that the measured fragment length histograms $\left\{W_{i}(\mathcal{H})\right\}_{i=1}^{N}$, where $N$ is the number of bins and $W_{i}$ is the number of nanotubes in bin $i$, were generated by independent identically distributed fragment samples from length distribution predicted by the model $\left\{W_{i}\left(L_{c r i t}\right)\right\}_{i=1}^{N}$. Then, $\operatorname{Prob}\left(\mathcal{H} \mid L_{\text {crit }}\right)$ can be conveniently calculated as likelihood: $\ln \operatorname{Prob}\left(\mathcal{H} \mid L_{\text {crit }}\right)=D+\Sigma_{i}\left[W_{i}(\mathcal{H}) \cdot \ln W_{i}\left(L_{\text {crit }}\right)\right]$, where $D$ is a constant independent of $L_{c r i t}$ and absorbed during normalization.

The fragment length distribution predicted by the scission model was computed from stochastic scission simulation of a large number of nanotubes $(>40,000)$ having the experimentally measured length distribution of the DNA nanotubes 
before passing through the microfluidic device. These DNA nanotubes were subjected to the following stochastic scission rules.

First, we note that the number of DNA nanotubes that crosses the orifice at position $(x, y)$ is proportional to the flow rate at the orifice $u_{w}(x, y)$. By solving the Navier-Stokes equation for incompressible flow in a rectangular channel, one can obtain an expression for the flow profile involving an infinite series,

$$
\begin{aligned}
u_{w}(x, y)= & E\left\{\left[x^{2}-\left(\frac{w}{2}\right)^{2}\right]\right. \\
& \left.+\sum_{n=o}^{n} \frac{8}{a} \frac{(-1)^{n}}{\alpha_{n}^{3}} \frac{\cosh \left(\alpha_{n} y\right)}{\cosh \left(\alpha_{n} h / 2\right)} \cos \left(\alpha_{n} x\right)\right\},
\end{aligned}
$$

where $\alpha_{n}=(2 n+1) \frac{\pi}{w}$ and $E$ is a constant obtained by setting $u_{w}(0,0)$ to be the maximum flow rate $u_{w}^{\max }$. In this coordinate system, $(x=0, y=0)$ is chosen to be the center of the channel where the maximum flow occurs and the range of width and height of the flow channel are $[-w / 2, w / 2]$ and $[-h / 2, h / 2]$, respectively. In our simulation, we use the normalized $u_{w}\left(x_{i}, y_{i}\right)$ as the probability distribution for stochastically assigning $\left(x_{i}, y_{i}\right)$ to nanotube $i$.

Second, the fragment size produced by scission of nanotube $i$ depends on $\left(x_{i}, y_{i}\right)$. Using the same reasoning as employed in the position-dependent flux and Eqs. (A10) and (A12), one obtains the following expression for the critical length at $\left(x_{i}, y_{i}\right)$ :

$$
\begin{aligned}
& \sqrt{u_{w}^{\max } / \ln \left[L_{c r i t}\left(x_{i}, y_{i}\right) /(2 R)\right]} L_{c r i t}\left(x_{i}, y_{i}\right) \\
& =\sqrt{u_{w}\left(x_{i}, y_{i}\right) / \ln \left[L_{c r i t} /(2 R)\right]} L_{c r i t} .
\end{aligned}
$$

In our model, an initial nanotube $i$ of length $L_{i}$ will experience a total of $m_{i}$ midpoint scission rounds, where $m_{i}$ is the largest non-negative integer that satisfies

$$
L_{c r i t}\left(x_{i}, y_{i}\right) \geq \frac{L_{i}}{2^{m_{i}}}
$$

From the equation above, $m_{i}$ will be given by $m_{i}$ $=\left\lfloor\ln \left[L_{i} / L_{\text {crit }}\left(x_{i}, y_{i}\right)\right] / \ln (2)\right\rfloor$, where the floor notation $\lfloor$.$\rfloor de-$ notes rounding down to the nearest integer. In our simulation, initial tube $i$ of length $L_{i}$ yields $2^{m_{i}}$ output fragments that have identical length of $L_{i} /\left(2^{m_{i}}\right)$. The simulation generated fragments were then tabulated to construct the fragment length distribution predicted by the scission model $\left\{W_{i}\left(L_{\text {crit }_{i}}\right)\right\}_{i=1}^{N}$ for computing $\operatorname{Prob}\left(L_{\text {crit }} \mid \mathcal{H}\right)$.

\section{APPENDIX C: BEST $L_{\text {crit }}$ FIT BY BAYESIAN INFERENCE}

Fragment length distributions for six-, eight-, and tenhelix nanotubes for volumetric flow rates with values given by $\sqrt{2^{n}} \mathrm{ml} / \mathrm{h}$, where $n$ is an integer in the range $-2 \leq n \leq 4$, are shown in Fig. 7. In each analysis, the fragment length measurement was stopped when the fragment counts reached $\approx 250$ fragments. The Bayesian inference of 250 simulated fragments with a chosen $L_{c r i t}$ shows robust results within $\pm 12 \%$ from the chosen $L_{c r i t}$ for $L_{c r i t}$ smaller than the mean of initial nanotube distribution. The blue dot with blue error bars represents the average fragment length for each run. The Bayesian analysis was performed by comparing our measurement with simulation using one adjustable parameter, namely, critical length $L_{c r i t}$, as described in the main text. The best simulated distribution by Bayesian a posteriori probability (orange line) fits our data fairly well. The orange circle denotes the most probable $L_{\text {crit }}$ in each experiment. The orange error bar spans the range where $\operatorname{Prob}\left(L_{\text {crit }} \mid \mathcal{H}\right)$ is over $90 \%$ based on our model.

\section{APPENDIX D: BEST $\boldsymbol{L}_{c r i t}$ FIT BY BAYESIAN INFERENCE WITH TRUNCATED GAUSSIAN NOISE}

Best $L_{\text {crit }}$ fit for the scission model with the addition of truncated Gaussian noise, summarized in Fig. 8, shows that adding noise to account for unmodeled physical source of randomness does not significantly improve the Bayesian fit. With the addition of noise, each scission event produces two not exactly equal fragment lengths. For nanotube $i$, the standard deviation of the truncated Gaussian noise $\sigma_{i}$ was chosen to be proportional to tube length $L_{i}$, reflecting the results of the induced drag force calculation for which the region where the tension reaches plateau becomes narrower as the nanotube tube gets shorter. We truncated the Gaussian noise at 0 and $L_{i}$ fragment sizes to eliminate unphysical fragment outputs in our simulation, namely, fragments with negative lengths and fragmented nanotubes longer than initial fragment length $L_{i}$. The Bayesian fit was performed over a wide range of model parameters $\left(0.02 L_{i}<\sigma_{i}<0.50 L_{i}, 0.05<L_{\text {crit }}\right.$ $<10.00)$. The upper bound of the $\sigma_{i}$ corresponds to substantially large noise such that for a nanotube length $L_{i}$, where $L_{i}>2 L_{\text {crit }}$, the probability of scission at any point along the fragment, including no scission at all, is approximately equal. Note also that the distribution of the truncated Gaussian with the upper bound of $\sigma_{i}$ is close to uniform distribution between 0 and $L_{i}$. The orange and red circles with error bars represent the best $L_{c r i t}$ fit by Bayesian inference for polymer scission without and with noise, respectively. Similarly, the orange and red lines are the best distribution fit to our normalized fragment length histogram based on simulation without and with noise, respectively.

The Bayesian histogram fits of the model with (red lines) and without noise (orange lines) show similar shapes (Fig. 8) and further support our simple scission model presented in the main text. The extracted $L_{c r i t}$ from the Bayesian inference with noise is consistent within $15 \%$ of the fit in the absence of noise. The agreement is within 5\% if we exclude the slowest volumetric flow-rate data where the inference has the widest $90 \%$ confidence bands. The maximum value of the most probable $\sigma_{i}$ overall fits is $0.20 L_{i}$. This value of $\sigma_{i}$ still represents truncated Gaussian noise distribution whose width is substantially smaller than the tube length $L_{i}$. 
[1] B. A. Buchholz, J. M. Zahn, M. Kenward, G. W. Slater, and A. E. Barron, Polymer 45, 1223 (2004).

[2] P. Oefner, S. Hunicke-Smith, L. Chiang, F. Dietrich, J. Mulligan, and R. Davis, Nucleic Acids Res. 24, 3879 (1996).

[3] Y. Thorstenson, S. P. Hunicke-Smith, P. J. Oefner, and R. D. Davis, Genome Res. 8, 848 (1998).

[4] M. Quail, in Encyclopedia of the Human Genome, edited by D. N. Cooper (Nature Publishing, London, 2003), Vol. 10.

[5] T. R. Serio, A. G. Cashikar, A. S. Kowal, G. J. Sawicki, J. J. Moslehi, L. Serpell, M. F. Arnsdorf, and S. L. Lindquist, Science 289, 1317 (2000).

[6] S. R. Collins, A. Douglass, R. D. Vale, and J. S. Weissman, PLoS Biol. 2, e321 (2004).

[7] A. F. Horn and E. W. Merrill, Nature (London) 312, 140 (1984).

[8] R. Bowman and N. Davidson, Biopolymers 11, 2601 (1972).

[9] B. Dancis, Biophys. J. 24, 489 (1978).

[10] J. Odell and A. Keller, J. Polym. Sci., Part B: Polym. Phys. 24, 1889 (1986).

[11] T. Q. Nguyen and H.-H. Kausch, Polymer 33, 2611 (1992).

[12] J. Odell and M. Taylor, Biopolymers 34, 1483 (1994).

[13] S. Vanapalli, S. Ceccio, and M. Solomon, Proc. Natl. Acad. Sci. U.S.A. 103, 16660 (2006).

[14] J. Larson et al., Lab Chip 6, 1187 (2006).

[15] P. Yin, R. F. Hariadi, S. Sahu, H. M. T. Choi, S. H. Park, T. H. LaBean, and J. H. Reif, Science 321, 824 (2008).

[16] R. Schulman and E. Winfree, Proc. Natl. Acad. Sci. U.S.A. 104, 15236 (2007).

[17] F. Oosawa and M. Kasai, J. Mol. Biol. 4, 10 (1962).

[18] S. B. Smith, Y. Cui, and C. Bustamante, Science 271, 795 (1996).

[19] E. Winfree, F. Liu, L. A. Wenzler, and N. C. Seeman, Nature (London) 394, 539 (1998).

[20] P. W. K. Rothemund, A. Ekani-Nkodo, N. Papadakis, A. Kumar, D. K. Fygenson, and E. Winfree, J. Am. Chem. Soc. 126, 16344 (2004).
[21] P. W. K. Rothemund, N. Papadakis, and E. Winfree, PLoS Biol. 2, e424 (2004).

[22] F. A. Aldaye, A. L. Palmer, and H. F. Sleiman, Science 321, 1795 (2008).

[23] R. H. Wade and D. Chrètien, J. Struct. Biol. 110, 1 (1993).

[24] A. Ekani-Nkodo, A. Kumar, and D. K. Fygenson, Phys. Rev. Lett. 93, 268301 (2004).

[25] T. Fu and N. Seeman, Biochemistry 32, 3211 (1993).

[26] V. Bloomfield, D. Crothers, and I. Tinoco, Nucleic Acids: Structures, Properties, and Functions (University Science Books, Sausalito, CA, 2000).

[27] B. Tinland, A. Pluen, J. Sturm, and G. Weill, Macromolecules 30, 5763 (1997).

[28] D. Duffy, J. McDonald, O. Schueller, and G. Whitesides, Anal. Chem. 70, 4974 (1998).

[29] M. Unger, H. Chou, T. Thorsen, A. Scherer, and S. Quake, Science 288, 113 (2000).

[30] I. Braslavsky, B. Hebert, E. Kartalov, and S. Quake, Proc. Natl. Acad. Sci. U.S.A. 100, 3960 (2003).

[31] See http://rsbweb.nih.gov/ij/

[32] R. G. Larson, T. T. Perkins, D. E. Smith, and S. Chu, Phys. Rev. E 55, 1794 (1997).

[33] J. Morfill, F. Kuhner, K. Blank, R. A. Lugmaier, J. Sedlmair, and H. E. Gaub, Biophys. J. 93, 2400 (2007).

[34] C. Bustamante, S. B. Smith, J. Liphardt, and D. Smith, Curr. Opin. Struct. Biol. 10, 279 (2000).

[35] C. Radding, K. Beattie, W. Holloman, and R. Wiegand, J. Mol. Biol. 116, 825 (1977).

[36] I. Panyutin and P. Hsieh, Proc. Natl. Acad. Sci. U.S.A. 91, 2021 (1994).

[37] B. Yurke and R. F. Hariadi (unpublished).

[38] R. Schulman and E. Winfree, Advances in Artificial Life (Springer-Verlag, Berlin, 2005), 734-743.

[39] K. D. Knudsen, J. G. H. Cilfre, and J. G. de la Torre, Macromolecules 29, 3603 (1996).

[40] T. Nguyen and H. Kausch, Macromolecules 23, 5137 (1990). 